\title{
Amplitude modulations and inter-trial phase stability of alpha-oscillations differentially reflect working memory constraints across the lifespan
}

\author{
Myriam C. Sander, Markus Werkle-Bergner *, Ulman Lindenberger \\ Max Planck Institute for Human Development, Berlin, Germany
}

\section{A R T I C L E I N F O}

Article history:

Received 23 March 2011

Revised 9 June 2011

Accepted 29 June 2011

Available online 7 July 2011

\section{Keywords:}

Aging

Alpha activity

Development

EEG

Visual working memory

\begin{abstract}
A B S T R A C T
Working memory (WM) capacity increases across childhood, peaks in young adulthood, and declines thereafter. Developmental and aging theories suggest that deficient inhibitory control processes in children and older adults may underlie the lower performance relative to younger adults. Recently, oscillatory alpha power $(7-13 \mathrm{~Hz})$ of the electroencephalogram (EEG) has been suggested as a neural marker of inhibition processes contributing to WM performance (Sauseng et al., 2009). We examined 20 children (10-13 years), 12 younger adults ( $20-26$ years), and 20 older adults ( $70-76$ years) in a cued change-detection paradigm. Behaviorally, we observed the expected lifespan peak in younger adults. EEG alpha power was generally reduced in older adults compared to children and younger adults. In line with previous research, hemispheric differences in alpha power related to attention and WM processes during the retention interval increased with load in younger adults. In children and older adults, lateralized alpha power increased from low to medium load conditions, but decreased for high load conditions. Furthermore, older adults showed higher inter-trial phase stability shortly after stimulus onset compared to children and younger adults. Our results show that inhibitory control processes as indexed by local alpha power modulations can be observed in children and older adults but seem to break down when WM load is high. In addition, older adults are more entrained by external stimulation what may increase a need for inhibitory control during later processing. We conclude that differences in inhibitory control processes and information uptake as reflected in amplitude modulations and inter-trial phase stability of alpha rhythms interactively determine WM constraints across the lifespan.
\end{abstract}

(c) 2011 Elsevier Inc. All rights reserved.

\section{Introduction}

An important aspect of visual working memory (WM) refers to the ability to temporarily maintain perceptual input of the environment in mind so that the information can be used for goal-directed behavior (D'Esposito, 2007). WM capacity is assumed to be limited (Cowan, 2011; Miller, 1956), with considerable variability of capacity estimates across individuals (Luck and Vogel, 1997; Vogel and Machizawa, 2004) and age groups (Cowan et al., 2006; Jost et al., 2011; Riggs et al., 2006; Sander et al., 2011a). WM performance is not only dependent on successful retention of relevant information but also on efficient suppression of irrelevant information (McNab and Klingberg, 2008; Vogel et al., 2005; Zanto and Gazzaley, 2009) during early and late stages of processing (Lavie et al., 2004; Zanto and Gazzaley, 2009). Vogel et al. (2005) recently provided evidence for a positive relation between individual differences in "filter efficiency" and WM capacity. Investigating event-related potentials (ERPs) in a cued change detection paradigm, they could show that individuals

\footnotetext{
* Corresponding author at: Center for Lifespan Psychology, Max Planck Institute for Human Development, Lentzeallee 94, 14195 Berlin, Germany. Fax: + 49308249939. E-mail address: werkle@mpib-berlin.mpg.de (M. Werkle-Bergner).
}

who are better able to prevent processing of irrelevant information have higher WM capacity. Conversely, individuals with lower WM capacity thus seem to keep irrelevant information in mind (see Jost et al., 2011, for a similar study including older adults).

Recently, Sauseng et al. (2009) studied the oscillatory neuronal substrates of WM using similar procedures as Vogel et al. (2005). They observed that the amplitude of alpha oscillations $(\sim 8-12 \mathrm{~Hz})$ during the retention interval was larger ipsilaterally than contralaterally to the attended hemifield. Importantly, this lateralized difference in alpha power increased with load and predicted individual WM capacity. In line with previous research, the results by Sauseng et al. (2009) point to the importance of alpha oscillations for inhibitory processing (Kelly et al., 2006; Rihs et al., 2007). In addition, these results suggest that inhibitory control mechanisms, WM performance, and alpha oscillations are intertwined (Jensen and Mazaheri, 2010).

Ontogenetically, WM performance shows an increase in childhood (Gathercole, 1999), a peak in young adulthood, and marked decline with advancing adult age (Park and Payer, 2006). Prominent theories of child development (Dempster, 1992) and cognitive aging (Hasher et al., 2007; Hasher and Zacks, 1988) relate this lifespan trajectory to the late maturation and early decline of the prefrontal lobes and the neural circuitry connecting the prefrontal lobes to more posterior 
parts of the brain (for child development, see Sowell et al., 1999, 2003; Toga et al., 2006; for adult development and aging, see Lindenberger et al., in press; Raz et al., 2005; Raz and Rodrigue, 2006). To some extent, then, age-related performance differences in WM may reflect less efficient inhibitory processing in children and older adults due to immature or senescing prefrontal lobes.

In line with these considerations, a recent study observed adult age differences in the suppression of irrelevant information (Gazzaley et al., 2008) as indicated by age group differences in early ERP components during WM encoding (see also Zanto et al., 2009). At the same time, Gazzaley et al. (2008) also found that later suppression processes as indicated by modulations of alpha band activity were relatively intact in older adults. The authors suggested that normal aging does not abolish inhibitory processes in WM, but rather delays them to later processing stages (see also Jost et al., 2011). Gazzaley et al. (2008) presented stimuli sequentially, which makes it difficult to distinguish between encoding and retention processes. Furthermore, WM load was rather low and held constant in that study. Based on the results of recent functional magnetic resonance imaging studies (e.g., Nagel et al., 2009; Schneider-Garces et al., 2010), it seems likely that age-related differences in alpha oscillations are more pronounced when WM load is high.

Child-developmental research on the role of alpha oscillations in memory tasks is scarce. One study (Gomarus et al., 2006) investigated effects of stimulus load and stimulus relevance in a visual memory task in children. In this study, the presentation of the memory array was followed by a sustained desynchronization of alpha power, which was more pronounced for larger memory load conditions (see also Ciesielski et al., 2010). Doesburg et al. (2010) recently investigated local alpha-band synchronization and long-range alpha phase synchronization during short-term memory retention in children. In this study, alpha amplitudes over posterior MEG sensors decreased during the early retention interval. Doesburg et al. (2010) suggested that this desynchronization reflects the task-dependent activation of the visual cortex. The authors furthermore observed increased alpha band synchronization between posterior sensors and widespread cortical regions, which was interpreted as a mechanism for maintaining memory traces. However, WM load was not varied in this study.

In sum, alpha oscillations have been reliably observed in children and older adults, and their age-related changes may relate to cognitive changes in childhood (Uhlhaas et al., 2010) and adulthood (Klimesch, 1999). However, to our knowledge, no study has investigated the relation between age group differences in WM capacity and alpha oscillations across the lifespan. Given the role of alpha oscillations in inhibitory processing, age differences in the task-dependent modulation of alpha band activity may contribute to age-related behavioral differences in WM performance.

We therefore asked children, younger, and older adults to perform a cued change detection task. In the typical change-detection paradigm, the observer is presented with a memory array of varying number of items (e.g., colored squares). After a short retention interval of about $1 \mathrm{~s}$, the participant has to compare the internally maintained representation of the memory array to a probe array in which one of the items has or has not been altered. By varying the number of presented items, this procedure yields an estimate of the individual WM capacity, the so-called $k$-score (Cowan, 2001). In the cued version of this task, a cue prior to the memory array instructs the participant to only maintain the items of the indicated hemifield, where changes may occur. Thus, beside the maintenance of the relevant information, this version of the task also requires the inhibition of information from the not-attended hemifield.

In the present study, load levels and presentation times were varied within subjects. Prior ERP analyses of this data set, which were reported elsewhere (Sander et al., 2011b), focused on age differences in the contralateral delay activity, a difference wave occurring during the maintenance period of the task (Vogel and Machizawa, 2004). In the present analyses, we focus on alpha power differences between ipsilateral and contralateral hemisphere relative to the attended hemifield during the retention interval, henceforth referred to as lateralized alpha power. Age differences in alpha frequency (Klimesch, 1999) were taken into account by basing the analyses on individual alpha frequencies (IAF). We assumed that lateralized alpha power would be less strong in children and older adults than in younger adults, especially at high WM load, reflecting less efficient inhibitory control operations in these two groups relative to younger adults. Furthermore, we investigated alpha inter-trial phase stability prior to the retention interval as an indicator of age differences in the control of early stimulus-evoked encoding processes (Freunberger et al., 2009). Based on previous research using event-related potentials (Gazzaley et al., 2008; Zanto et al., 2009), we predicted that older adults might also show early selection deficits in the timefrequency domain.

\section{Material and methods}

\section{Participants}

Given that interindividual variation is usually larger in children and older adults relative to younger adults, we aimed at larger sample sizes for these two age groups. The effective sample comprised 20 children $\left(M_{\text {age }}=11.91, S D_{\text {age }}=.53\right.$, range $10-13$ years $), 12$ younger adults $\left(M_{\text {age }}=24.19, S D_{\text {age }}=1.57\right.$, range $20-26$ years $)$, and 20 older adults $\left(M_{\text {age }}=73.30, S D_{\text {age }}=1.58\right.$, range $70-76$ years $)$.

The original sample included 31 children, 19 younger adults, and 31 older adults. From this sample, we excluded participants that showed excessive EEG artifacts due to muscle activity or eye movements. Furthermore, we excluded 1 older adult because behavioral performance was at chance levels. Compared to our analysis of the ERP data (Sander et al., 2011b), the sample was further reduced in this study because resting state data was not available for 2 children and 2 older adults due to technical problems.

To document the age typicality of the sample, all participants were assessed on tests of verbal knowledge (Spot-a-Word; cf. Lehrl, 1977) and perceptual speed (digit symbol substitution test, cf. Wechsler, 1955) as well as on a test of sustained attention (d2; Brickenkamp, 1994). In addition, visual acuity was measured in Snellen decimal units at two different distances $(30 \mathrm{~cm}$ and $5 \mathrm{~m}$ ) using Landolt rings (Geigy, 1977). Table 1 displays sample descriptives. The ethics committee of the Max Planck Institute for Human Development, Berlin, approved the study.

\section{Experimental paradigm}

During the experimental procedure, participants were seated comfortably in a dimly lit as well as electromagnetically and acoustically shielded room. The EEG measurement started with a 6minute relaxation phase (resting EEG, 3 min with eyes closed and 3 min with eyes open). Relaxation served to adapt the participants to the lab environment and was followed by the task. A hemifield version of the change-detection task (Vogel and Machizawa, 2004)

\section{Table 1}

Descriptive summary of covariate measures.

\begin{tabular}{|c|c|c|c|}
\hline \multirow[t]{2}{*}{ Measure } & $\begin{array}{l}\text { Children } \\
(\mathrm{n}=20)\end{array}$ & $\begin{array}{l}\text { Young adults } \\
(\mathrm{n}=12)\end{array}$ & $\begin{array}{l}\text { Older adults } \\
(\mathrm{n}=20)\end{array}$ \\
\hline & $\mathrm{M}(\mathrm{SD})$ & $\mathrm{M}(\mathrm{SD})$ & $\mathrm{M}(\mathrm{SD})$ \\
\hline Digit symbol & $50.75(7.35)$ & $66.92(10.67)$ & $50.52(9.38)$ \\
\hline Vocabulary & $17.20(3.49)$ & 24.17 (3.69) & $29.14(3.18)$ \\
\hline Close vision & $.86(.13)$ & $.82(.12)$ & $.42(.14)$ \\
\hline Far vision & $1.22(.44)$ & $1.39(.55)$ & $1.01(.34)$ \\
\hline
\end{tabular}


was used to test the hypotheses (see Fig. 1). Memory arrays of colored squares were presented in four blocks to the participants. Presentation times of the memory array were set to $100 \mathrm{~ms}$ and $500 \mathrm{~ms}$ in two blocks each. The first two and the last two blocks were always presented at the same presentation time, and the order of the presentation time was counterbalanced across participants. Targets were defined as squares presented in one hemifield as indicated by a centrally placed cue before each trial. To keep the difficulty of the task comparable for the different age groups, we presented memory arrays of 2,4 , or 5 targets to younger adults and memory arrays of 2,3 or 4 targets to older adults and children. In the following, we will refer to these set sizes as representing low, medium and high levels of WM load. Set sizes were randomized within blocks. After a retention interval of $1000 \mathrm{~ms}$, a probe array of colored squares was shown and participants had to indicate whether all the colors of the probe array's targets were identical to the memory array or whether one of the squares had changed in color. Subjects answered by pressing buttons labeled as "same" (in German: "gleich") or "different" ("ungleich"). The mapping on response alternative on participants' hands was counterbalanced across individuals. Participants were encouraged to respond as accurately as possible and to guess if they were not sure of their response. Maximum response time was restricted to $5000 \mathrm{~ms}$. Each block started with 12 practice trials to allow participants to get used to the current presentation time. Afterwards, each participant completed 360 trials of varying set size per presentation time. Set sizes were randomized within blocks. After each block, participants got feedback about the accuracy of their responses.

Given that we assumed older adults and children having additional difficulties with a cued hemifield presentation, we always presented the cue for $500 \mathrm{~ms}$ and showed it until the memory array was presented to minimize cue-related memory load. We also blocked the cue direction for 30 consecutive trials to prevent a task-switching situation that would differentially affect the age-groups (e.g., Davidson et al., 2006; Kray and Lindenberger, 2000).

\section{Stimuli}

Stimulus presentation and recording of behavioral responses were controlled with Eprime v1.2 software (Psychology Software Tools, Inc., Pittsburgh, PA, USA). Stimuli consisted of colored squares $\left(0.65^{\circ} \times 0.65^{\circ}\right.$ of visual angle) presented on gray background (RGB values: $200,200,200$ ) within an area of $4^{\circ} \times 7.3^{\circ}$ of visual angle right and left of the fixation cross (distance to the fixation cross was $1.5^{\circ}$ ). Spatial locations of the squares were random, with a minimum distance of $2^{\circ}$ between the centers of the squares. Participants sat at a viewing distance of $70 \mathrm{~cm}$. Colors were randomly selected from a set of 11 highly discriminable values, black (RGB values: $0,0,0$ ), white (RGB: 255, 255, 255), gray (RGB: 126, 123, 126) blue (RGB: 0, 0, 255), green (RGB values: 0, 255, 0), red (RGB: 255, 0, 0), cyan (RGB: 0, 255, 255 ), violet (RGB: 255, 0, 255), brown (RGB: 153, 102, 51), orange (RGB: $255,112,1$ ), and yellow (RGB: $255,255,0$ ). The same color was not repeated more than twice per array. Pilot testing indicated that some color changes were difficult to detect for older adults. Hence, we excluded changes from black to blue or gray, from blue to black or gray, from red to orange, brown or magenta, from magenta to red,

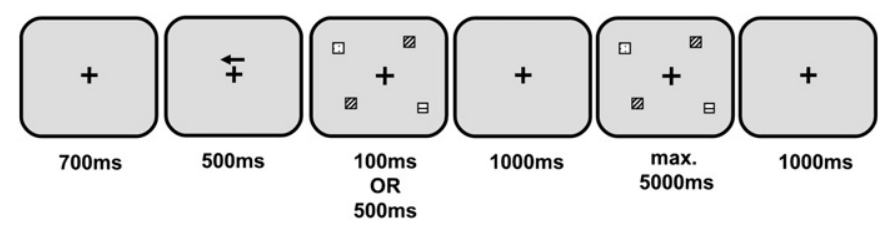

Fig. 1. Experimental paradigm. Different patterns of the squares represent different colors. from brown to red or orange, from orange to red or brown and from gray to black or blue.

\section{EEG recording and preprocessing}

EEG was recorded continuously with BrainVision amplifiers (BrainVision Products $\mathrm{GmbH}$, Gilching, Germany) from $61 \mathrm{Ag} / \mathrm{Ag}-\mathrm{Cl}$ electrodes embedded in an elastic cap. Three additional electrodes were placed at the outer canthi (horizontal EOG) and below the left eye (vertical EOG) to monitor eye movements. During recording, all electrodes were referenced to the right mastoid electrode, while the left mastoid electrode was recorded as an additional channel. Electrode impedances were maintained below $5 \mathrm{k} \Omega$ before recordings. The EEG was recorded with a pass-band of 0.1 to $250 \mathrm{~Hz}$ and digitized with a sampling rate of $1000 \mathrm{~Hz}$.

For preprocessing, the EEG was re-referenced to mathematically linked mastoids, downsampled to $256 \mathrm{~Hz}$, and band-pass filtered between $0.5 \mathrm{~Hz}$ and $125 \mathrm{~Hz}$. Four-second data epochs were extracted from $-2 \mathrm{~s}$ to $2 \mathrm{~s}$ with respect to the memory array onset. The extracted segments were visually inspected for eye movements and excessive muscle activity. Trials with eye movements (especially saccades) and muscle activity were rejected from analysis. Afterwards, an independent-component analysis (ICA) was used to correct for remaining eye blink, noise, and muscle activity (Jung et al., 2000). ICs representing artifactual sources were visually identified and removed from the data.

\section{Data analysis}

Previous studies have examined load modulations of lateralized alpha power activity at $100 \mathrm{~ms}$ presentation times (Sauseng et al., 2009). Therefore, the analyses presented in this study are focused on this presentation time condition, which is the standard condition used in change detection paradigms. ${ }^{1}$

\section{Behavioral analysis}

Behavioral performance as a function of age group and task load was assessed with the $\mathrm{Pr}$-score (i.e., hit rate minus false alarm rate). In addition, we also calculated the $k$-score for each set size [i.e., $k=$ (hit rate - false alarm rate) $\times$ set size; cf. Cowan, 2001]. Generally, $k$ is assumed to increase with increasing set size until it reaches an asymptote at the individual maximum performance level. To characterize age group differences in WM performance, we compared the maximum $k$-score between age groups. Note that maximum $k$ scores were achieved under different load conditions in the different age groups and should therefore be compared with caution.

\section{Electrophysiological analysis}

All analyses were performed with the Fieldtrip software package (developed at the F. C. Donders Centre for Cognitive Neuroimaging, Nijmegen, The Netherlands; http://fieldtrip.fcdonders.nl/) supplemented with custom-made MATLAB code (The MathWorks Inc., Natick, MA, USA).

To determine the individual alpha peak frequencies (IAFs), power spectra were computed for the eyes-closed resting state data $(+0.5 \mathrm{~s}$ to $+2.5 \mathrm{~s}$ ) by means of fast-Fourier transformations (FFT) and averaged across all occipito-parietal electrodes $(\mathrm{Oz}, \mathrm{Pz}, \mathrm{POz}, \mathrm{O} 1 / 2$, $\mathrm{PO} 3 / 4, \mathrm{PO} 7 / 8, \mathrm{P} 1 / 2, \mathrm{P} 3 / 4, \mathrm{P} 5 / 6, \mathrm{P} 7 / 8)$. The IAF was then defined as the maximum peak of the averaged power-spectra between 7 and $13 \mathrm{~Hz}$. IAF distributions for each age group are presented in Fig. 2.

\footnotetext{
${ }^{1}$ In exploratory analyses we tried to identify load dependent lateralized alpha power also in the condition of $500 \mathrm{~ms}$ presentation time. Given that we did not observe the effect of interest in our reference group of younger adults, we refrained from further analyses of that condition.
} 


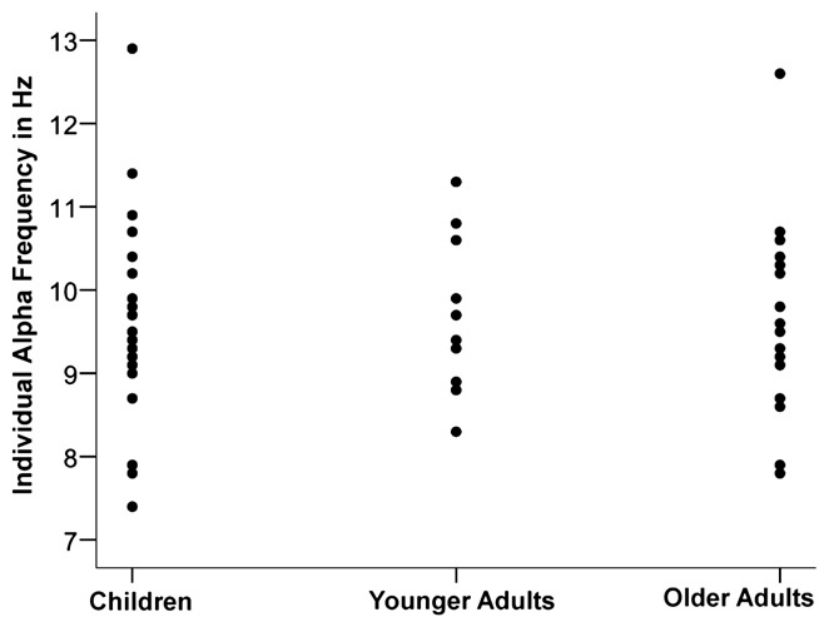

Fig. 2. Distributions of individual alpha frequencies separately for each age group.

With regard to the task, ICA-cleaned, artifact-free EEG trials with correct responses were analyzed. To extract oscillatory power and phase from $4 \mathrm{~Hz}$ to $20 \mathrm{~Hz}$ in steps of $1 \mathrm{~Hz}$, the amplitude time-series of each single trial at each electrode was convolved with a set of Morletwavelets (Tallon-Baudry et al., 1997) with a cycle length of 7. The timevarying power of the signal for each center frequency is given by the squared norm of the respective complex-valued wavelet-coefficients. The time-varying phase is given by computing the inverse tangens of the ratio of the real and imaginary parts of the wavelet-coefficients.

For the analysis of alpha power, we collapsed data across all load conditions and defined successively frequency of interest, time window of interest, and electrode of interest. We defined the IAF $+/-1 \mathrm{~Hz}$ as frequency of interest at the individual level. In line with previous research we defined the time window of interest (TOI) from 400 to $900 \mathrm{~ms}$ after stimulus onset, covering most part of the retention interval, but excluding evoked activity due to the stimulus presentation. We then calculated the hemispheric difference in alpha power, that is, ipsilateral power minus contralateral power, and searched for the maximum hemispheric difference over posterior electrodes $(01 / 2, \mathrm{PO} / 4, \mathrm{PO} 7 / 8, \mathrm{P} 1 / 2, \mathrm{P} 3 / 4, \mathrm{P} 5 / 6, \mathrm{P} 7 / 8)$ on the group level across load conditions. The selection of the electrode cluster to determine the EOI was chosen based on the results of prior studies, according to which the peak of ipsi-contra differences is found over parietal regions (Sauseng et al., 2009). Electrodes of interest (EOI) were chosen as electrodes showing the largest effects: PO7/PO8 in younger adults, P7/P8 in older adults and PO3/PO4 in children (see Fig. 3). Age differences in load-modulated lateralized alpha power during the retention interval were investigated by extracting the

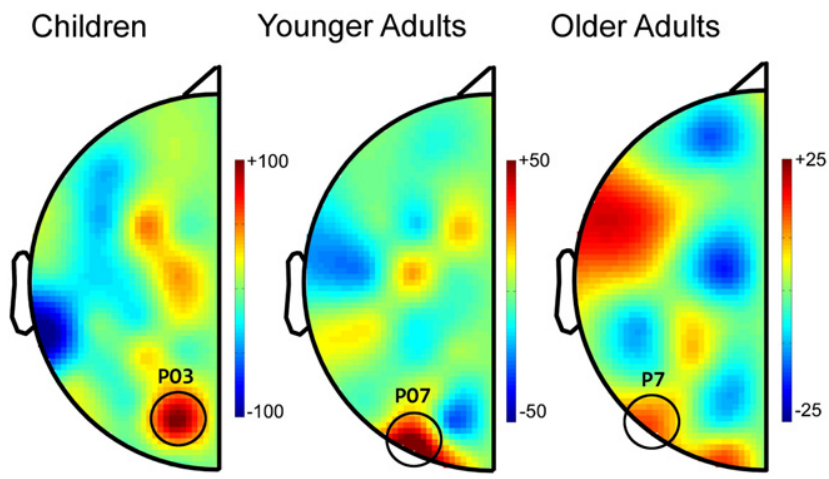

Fig. 3. Topographic distribution of alpha power differences during the retention interval across all load conditions. The electrode of interest used for later analyses is highlighted in black. Note the difference in scaling between age groups. mean power across the TOI at the EOI for the individual alpha frequency range.

The inter-trial phase stability of ongoing oscillations across trials was assessed with the phase-locking factor (PLF; see Tallon-Baudry et al., 1996), which was calculated as follows. First, the norm of the resultant mean direction vector was estimated across trials for each subject, electrode, and time-frequency point, separately for each load condition and cue direction. It has been shown that the number of trials used for analysis influences PLF estimates (Vinck et al., 2010). Therefore, we estimated the standard error for the PLF at each time-frequency point via a jackknife procedure (see e.g., Werkle-Bergner et al., 2009 for similar procedure). The estimated standard errors were used to derive $\mathrm{t}$-values with the number of degrees of freedoms (df) corresponding to the number of trials. The resulting t-values were transformed into $\mathrm{z}$ values (Hughett, 2007). A larger z-value then represents increased phase stability across trials. The z-values were averaged over lateral electrodes according to the cue direction resulting in ipsilateral and contralateral estimates of inter-trial phase stability. For analyses at the group level, we extracted the z-values for the corresponding IAF and EOI as determined from the analyses of oscillatory power. To analyze stimulus-evoked inter-trial phase stability, we determined the maximum phase stability after stimulus presentation in all age groups separately for the averaged z-values across participants and load conditions. Children showed maximum inter-trial phase stability at around $240 \mathrm{~ms}$, younger adults at $228 \mathrm{~ms}$ and older adults at $180 \mathrm{~ms}$ (see Fig. 4). For statistical comparisons, we extracted the mean of the $z$-values separately for each load condition in time windows of $+/-30 \mathrm{~ms}$ of maximum inter-trial phase stability.

\section{Statistical analysis}

Main analyses of age differences in performance, alpha power, and inter-trial phase stability were conducted with the Proc Mixed Procedure in SAS (SAS 9.1 for Windows). The ProcMixed model allows for heterogeneous variance and covariance structures across age groups and conditions in a repeated measures design. To constrain the data as little as possible, we fitted an unstructured covariance matrix to the data (for a similar approach in lifespan research see Shing et al., 2008; Werkle-Bergner et al., 2009). One-way ANOVA comparing age groups with regard to IAF and maximum $k$-scores was conducted with SPSS 15.0 for Windows (SPSS Inc., 1989-2006). The alpha level for all statistical analyses was set to $\alpha=.05$. Effect sizes are indicated by reporting the intraclass correlation coefficient $\rho_{I}$ or Cohen's $d$.

\section{Results}

\section{Behavioral markers of working memory capacity}

To keep task difficulty comparable across age groups, we used different load conditions in children and older adults than in younger

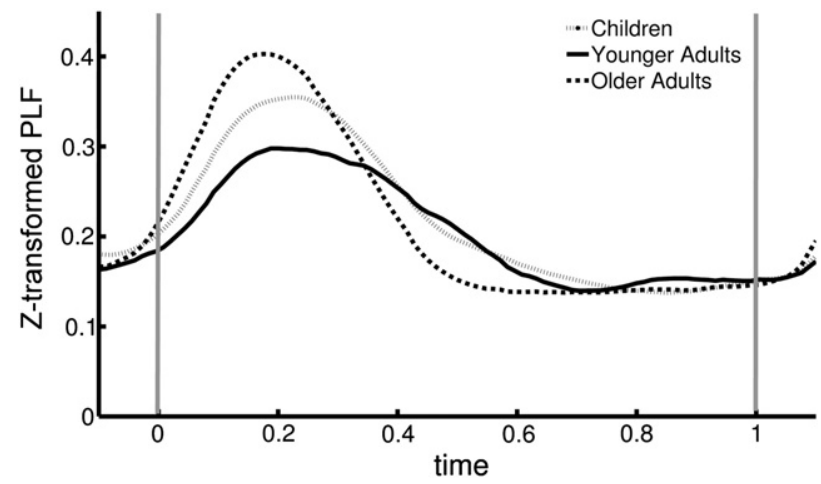

Fig. 4. Time-varying grand-average of PLF z-values. The vertical gray lines indicate stimulus onset and probe onset, respectively. 
adults. Therefore, in a first step, we conducted an overall mixed model on performance, calculated as $\mathrm{Pr}$-score $=$ hit rate - false alarm rate, with AGE GROUP (3) as between-subject factor and LOAD (3) as withinsubject factor to test whether this adaptation was successful. There was a main effect of $\operatorname{LOAD}, F(2,31)=174.96, p<.05, \rho_{I}=.96$, indicating that performance decreased with increasing memory load. Contrasts indicated that performance varied reliably by load; for low load versus medium load, $t(25.5)=12.85, p<.05, d=2.28$; for medium versus high load, $t(36.9)=8.33, p<.05, d=1.06$. Importantly, there was no main effect of AGE GROUP, $F(2,36.4)=.23, p>.8$, thus overall performance in the task did not differ between age groups. Nevertheless, we observed a significant interaction between AGE GROUP and LOAD, $F(4,35.8)=3.45$, $p<.05, \rho_{I}=.53$. Contrasts showed that this interaction was due to lower performance of children and older adults relative to younger adults in the low load condition; for children versus younger adults, $t(31.4)=$ $2.43, p<.05, d=.76$; for older adults versus younger adults, $t(31.4)=$ $2.43, p<.05, d=.81$, respectively. In medium and high load conditions, the three age groups did not differ, all $t<1.47$ and all $p>$.15. The finding that children and older adults did not show close-to-perfect performance under low load conditions is consistent with previous results from our lab (Sander et al., 2011a) and probably reflects age-related differences in the ability to sustain attention during the task. Means and standard errors of behavioral performance are displayed in Table 2.

In a second step we aimed at characterizing WM capacity differences between age groups by comparing the maximum $k$-score estimates. The $k$-score takes into account that comparable levels of performance are achieved under conditions of different loads in the three age groups. Using ANOVA, we found a main effect of AGE GROUP, $F(2,49)=6.00, p<.05, \rho_{I}=.44$, that was driven by higher $k$-scores in the group of younger adults $(M=2.89, S E=.21)$ compared to children $(M=2.34, S E=.10), t(30)=2.69, p<.05, d=.93$, and older adults $(M=2.20, S E=.11), t(30)=3.38, p<.05, d=1.14$. Children and older adults did not differ from each other, $t(38)=.80$, $p>.05$.

\section{Individual alpha frequencies}

Mean IAFs were $9.53(S E=.31)$ in children, $9.75(S E=.26)$ in younger adults, and $9.52(S E=.25)$ in older adults. One-way ANOVA indicated that age groups did not differ with regard to mean IAF, $F(2,49)=.174, p>.84$. A one-way ANOVA of resting power showed a main effect of AGE GROUP, $F(2,49)=4.853, p<.05, \rho_{I}=.41$. Contrasts showed that alpha resting power was significantly lower in older adults $(M=1.00, S E=.28)$ than in children $(M=2.73, S E=.51), t(38)=2.67$, $p<.05, d=.94$, and younger adults $(M=2.94, S E=.76), t(30)=2.60$, $p<.05, d=1.03$, whereas children and younger adults did not differ, $t(30)=.28, p>.05$.

\section{Power of alpha oscillations during the retention interval}

Alpha amplitudes of the retention interval were subjected to an overall mixed model with AGE GROUP as between-subject factor (3), and HEMISPHERE (2) and LOAD (3) as within-subjects factors. We found a main effect of AGE GROUP, $F(2,27.7)=13.07, p<.05, \rho_{I}=.70$. Contrasts showed that older adults had lower alpha power

Table 2

Behavioral performance ( $P r$-score $=$ hit rate - false alarm rate) separated for load condition and age group.

\begin{tabular}{llll}
\hline Load & $\begin{array}{l}\text { Children } \\
(\mathrm{n}=20)\end{array}$ & $\begin{array}{l}\text { Young adults } \\
(\mathrm{n}=12)\end{array}$ & $\begin{array}{l}\text { Older adults } \\
(\mathrm{n}=20)\end{array}$ \\
\cline { 2 - 3 } & $\mathrm{M}(\mathrm{SE})$ & $\mathrm{M}(\mathrm{SE})$ & $\mathrm{M}(\mathrm{SE})$ \\
\hline Low & $.86(.02)$ & $.93(.02)$ & $.87(.02)$ \\
Medium & $.71(.03)$ & $.62(.05)$ & $.67(.03)$ \\
High & $.55(.03)$ & $.55(.05)$ & $.52(.03)$ \\
\hline
\end{tabular}

$(M=347.03, S E=66.58)$ than children $(M=1541.31, S E=267.14)$ and younger adults $(M=1110.97, S E=280.48), t(22.5)=4.45, p<.05$, $d=1.37$ and $t(13.4)=2.77, p<.05, d=1.20$, respectively, whereas children and younger adults did not differ, $t(29.5)=1.15, p>.25$. There was also a main effect of HEMISPHERE, $F(1,32.6)=8.38, p<.05$, $\rho_{I}=.45$, with larger alpha power at ipsilateral $(M=1014.77$, $S E=135.41)$ than at contralateral sites $(M=984.77, S E=126.68)$. Also, the expected interaction between HEMISPHERE and LOAD, $F(2$, $32.1)=5.22, p<.05, \rho_{I}=.50$ was reliable. Contrasts revealed that hemispheric differences in alpha power were not detectable for small load conditions, $t(28.1)=0.11, p>.91$, but for medium, $t(31.5)=3.24$, $p<.05, d=.08$, and large load levels, $t(23.9)=2.75, p<.05, d=.04$. The interaction of AGE GROUP and HEMISPHERE showed a trend with $F(2,15.5)=3.15, p<.10, \rho_{I}=.54$, and, more importantly, the threeway interaction of AGE GROUP, HEMISPHERE and LOAD reached significance, $F(4,18.9)=2.87, p=.05, \rho_{I}=.61$. Given our a priori hypotheses, we conducted planned contrasts within each age group for the hemispheric difference at the different load conditions. In younger adults, ipsi- and contralateral activity did not differ for low load conditions, $t(12)=-.16, p>.87$, revealed a trend for medium load conditions, $t(12)=2.04, p<.10, d=.06$, and were significantly different for high load levels, $t(12)=2.45, p<.05, d=.07$. In children and older adults, only medium WM demands revealed significant differences between ipsilateral and contralateral activity, $t(20)=$ $2.27, p<.05, d=.09$ and $t(20)=2.14, p<.05, d=.05$. All other $p s>.15$ and $F s<1.47$. Results are illustrated in Fig. 5.

\section{Inter-trial phase stability of alpha oscillations after stimulus presentation}

In accordance with the analysis of alpha power, measures of intertrial phase stability were subjected to an overall mixed model with AGE GROUP (3) as between-subject factor and HEMISPHERE (2) and LOAD (3) as within-subject factors. This analysis yielded only a main effect of AGE GROUP, $F(2,34.5)=4.14, p<.05, \rho_{I}=.44$. Contrasts revealed that older adults $(M=4.03, S E=.29)$ showed higher inter-trial phase stability than younger adults $(M=2.86, S E=.34), t(27.7)=2.68$, $p<.05, d=.95$, and children $(M=3.18, S E=.24), t(38.4)=2.28$, $p<.05, d=.72$, whereas the children and younger adults did not differ, $t(23.6)=-.81, p>.42$. For all remaining main effects and interactions ps $>.06$ and $F s<2.3$.

\section{Discussion}

The present study investigated oscillatory neural correlates of WM selection processes in children, younger adults, and older adults. Behavioral markers revealed the expected differences in WM capacity between age groups, with children and older adults showing lower estimates compared to younger adults. The strength of alpha oscillations was generally reduced in older adults compared to younger adults and children. Most importantly, hemispheric differences in oscillatory power were differentially modulated by WM demands in the three age groups: In younger adults, hemispheric differences in alpha amplitudes were present at medium and high load. In contrast, children and older adults showed an inverted U-shape relation between alpha lateralization and load; lateralization of alpha oscillations increased from low to medium load, and declined from medium to high load. In addition, older adults exhibited stronger inter-trial phase stability immediately after stimulus presentation than younger adults and children.

\section{Age differences in task-related amplitude modulations of alpha oscillations during WM retention}

In line with previous studies that reported lower alpha amplitudes in older than younger adults (Müller et al., 2009; for a review see Klimesch, 1999), both resting and task-related alpha amplitudes were 

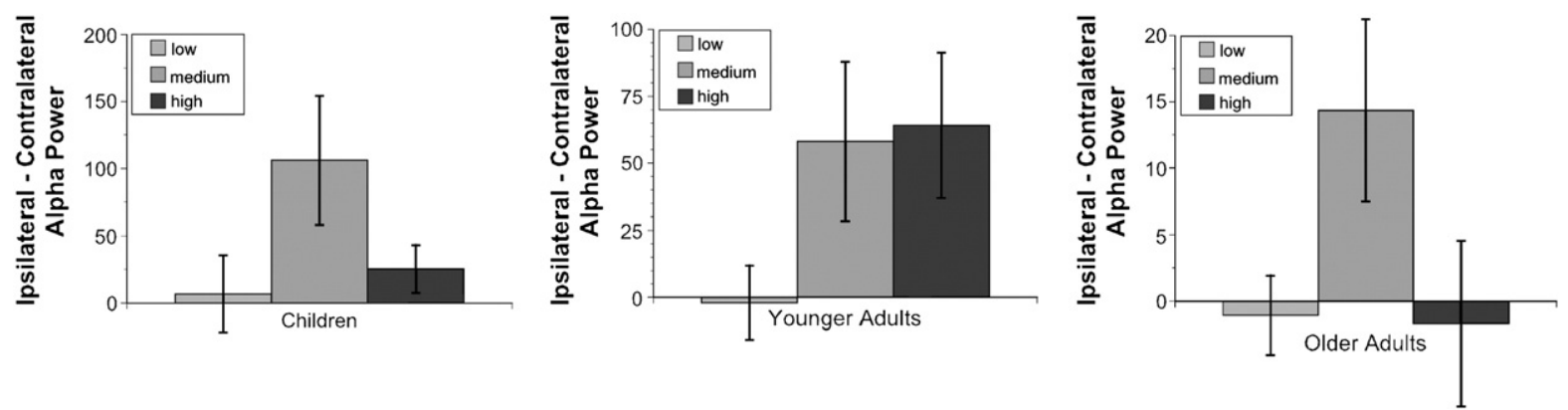

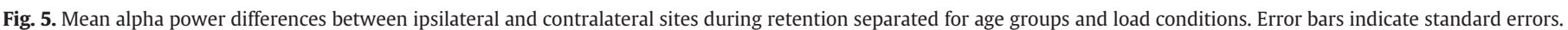
Note that due to general age differences in alpha power, scales differ between the plots for the different age groups.

smaller in older adults than in children and younger adults. However, general amplitude differences between age groups can hardly be interpreted unambiguously since they might be related also to age differences in brain size, brain geometry, or skull thickness (Frodl et al., 2001) and not to differences in cognitive processing per se. In contrast, decreased alpha amplitude during the delay interval in older adults in our study cannot be solely attributed to smaller tonic power because (a) the computation of difference scores between hemispheres effectively controls for general age differences in tonic alpha power; (b) alpha power during the delay interval varied by memory. Therefore, the modulation of alpha amplitudes by cognitive demands provides stronger evidence for understanding age differences in the functionality of alpha oscillations than age main effects (for similar arguments see Segalowitz and Davies, 2004; Werkle-Bergner et al., 2009).

Studies with samples of young adults have established an association between alpha oscillations and WM performance. Several studies observed an increase in alpha amplitudes over task-irrelevant regions during the retention interval with increasing load (e.g., Jensen et al., 2002; Tuladhar et al., 2007). Amplitude modulations of alpha oscillations seem to contribute to WM performance by inhibiting irrelevant information to prevent interference from conflicting stimuli (Freunberger et al., 2011; Klimesch et al., 2007b). The observation that alpha amplitudes are generally higher on sites not involved in target processing (Kelly et al., 2006; Rihs et al., 2007; Thut et al., 2006; Worden et al., 2000) further strengthens the assumption that alpha oscillations are associated with inhibitory control during visual spatial attention (Freunberger et al., 2011).

In the present study, we observed attention-related hemispheric differences in alpha amplitudes in all three age groups. In line with findings by Sauseng et al. (2009), younger adults showed a positive relation between alpha lateralization and WM load; ipsilateral alpha power was higher than contralateral power at medium and high loads, but not at low load. Note that the number of irrelevant items in the not-attended hemifield also increased with load. The present experimental design cannot disambiguate the relative contribution of relevant items and distracters. However, to disentangle the effects, Sauseng et al. (2009) performed a control experiment in which the number of targets and distracters was varied independently. The authors provided convincing evidence that lateralized alpha amplitudes vary with the number of distracters, and not with the number of targets. Based on their results and the theorizing of others (Freunberger et al., 2011; Jensen and Mazaheri, 2010; Klimesch et al., 2007a,b), we interpret the positive association between alpha lateralization and WM load in younger adults as a signature of increasing inhibitory control.

Interestingly, children and older adults showed a strikingly different pattern. Here, the modulation of lateralized alpha followed an inverted-U shape. Similar to younger adults, children and older adults did not show hemispheric differences in alpha power when WM demands were low, whereas lateralization was present at medium load conditions. However, when load levels were high and likely exceeded the WM capacity of children and older adults, the hemispheric differences in alpha power in these two age groups reverted to the level observed with low load.

This finding is important in two ways. First, it has not been known so far whether lateralized alpha modulation during spatial visual attention can be identified in children and older adults. Given that the hemispheric difference in alpha power can be interpreted as an indicator of attentional selection processes (Thut et al., 2006), the present study shows for the first time that both children and older adults are, in principle, able to attend to only one hemifield while suppressing distracting information in the irrelevant hemifield.

Second, lateralized alpha power was highest for the condition in which WM capacity was challenged, but not overtaxed. Given that the behavioral measures yielded mean estimates of about 2.3 items in children and 2.2 items in older adults, memory arrays with 3 items arguably were the appropriate load condition for these two age groups. Pr-Scores showed a significant decline in performance for high load conditions compared to medium load conditions. Hence, the decrease in alpha lateralization from medium to high load condition suggests that inhibitory control contributing to WM performance breaks down in children and older adults when task difficulty is too high. In line with this reasoning, Rissman et al. (2009) recently showed that participants failed to inhibit irrelevant distracters and did not show reliable signatures of neural suppression when WM load was high, whereas neural signatures of enhancement were not influenced by the WM load manipulation. The authors suggested that distracter filtering requires active cognitive control that cannot be exerted when WM capacity is taxed beyond optimal levels.

Though younger adults showed a comparatively reduced mean performance level under high load conditions as children and older adults, they continue to show reliable alpha power lateralization. Recent evidence suggests that neural correlates of WM do not differ between age groups when accounting for task difficulty (SchneiderGarces et al., 2010). Our results suggest that individuals of different ages recruit similar neural mechanisms when performance levels are calibrated, with the notable exception that younger adults' neural mechanisms may be more robust than children's and older adults' when taxed at exceedingly high levels of task difficulty.

\section{Age differences in alpha inter-trial phase stability after stimulus presentation}

Recent studies also noted early selection deficits in older adults using ERP measures (Gazzaley et al., 2008; Zanto et al., 2009). We examined the inter-trial phase stability of oscillatory activity shortly after stimulus presentation as an indicator of early perceptual encoding processes. In younger adults, higher phase stability has been found to be associated with better performance in perceptual (Hanslmayr et al., 2005) and memory (Klimesch et al., 2004) tasks. Accordingly, it has been suggested that high inter-trial phase stability 
reflects the efficient temporal coordination of cortical activation processes (Klimesch et al., 2007a; Sauseng and Klimesch, 2008).

In the present study, we observed increased inter-trial phase stability in all three age groups $180-240 \mathrm{~ms}$ after stimulus onset. Children and younger adults did not differ with regard to the amount of inter-trial phase stability. In line with a previous study (Yordanova and Kolev, 1997), this finding suggests that at the age of 10 to 13 years, the processing and transmission of visuo-spatial information are relatively mature. At the same time, inter-trial phase stability was actually higher in older adults than in children and younger adults. Given that several aging theories postulate a general decrease in the signal-to-noise ratio of neural processing with advancing adult age ( $\mathrm{Li}$ et al., 2006; MacDonald et al., 2006), the finding that older adults show higher inter-trial stability of neuronal oscillations, than younger adults and children may seem counterintuitive. However, recent studies reported similar evidence. For example, in an auditory oddball task, Müller et al. (2009) found that phase locking was higher in older adults than in younger adults. This effect was particularly pronounced during the first $200 \mathrm{~ms}$ after stimulus onset. Independent measures of perceptual speed showed a positive correlation to phase stability in younger adults, but a negative correlation in older adults. Similarly, in a recent study from our own lab (Werkle-Bergner et al., 2010), we observed that inter-trial phase stability was higher in to-beremembered items than in not-to-be-remembered items in a Sternberg WM-task (see Freunberger et al., 2009, for similar results in young adults). The preferential encoding of to-be-remembered items, presumably indicated by higher phase stability, predicted WM performance in younger adults and high-performing older adults, but not in low-performing older adults, again pointing to the possibility that high levels of phase locking may tax a different process in advanced old age than in childhood and in earlier periods of adulthood.

Based on these considerations, we suggest that stimulus-evoked inter-trial phase stability of oscillatory activity may change its meaning in the transition from early to late adulthood. The high levels of inter-trial phase stability of the older adults in the present and related studies may reflect a loss of complexity in the neurophysiological response (Cantero et al., 2009; cf. Thaler, 2002), that is, the response becomes more dependent on external stimulation. According to this line of thought, the inability of older adults to resist neural entrainment by the visual stimulus may underlie the early inhibition deficit in older adults, observed in other studies (Gazzaley et al., 2008; Zanto et al., 2010). Zanto et al. (2010) recently reported that in both attended and ignored conditions P1 amplitudes were enhanced in older adults compared to a passive viewing condition. Although the enhancement was larger in the attend condition, this result suggests that the inability to inhibit irrelevant information may result in inadvertent encoding of that information. In line with this interpretation, in a recent fMRI study, Schmitz et al. (2010) found that age-related impairments in perceptual attention may already be present at the level of the receptive field. They observed that older adults unintentionally co-encoded irrelevant information together with the relevant information, and maintained it in memory (cf. Campbell et al., 2010). In sum, we suggest that increased inter-trial phase stability after the stimulus in older adults may indicate less differentiated processing at the level of stimulus encoding (Park et al., 2004) due to senescent changes in neuronal architecture and/or neuromodulatory mechanisms (e.g. Backman et al., 2000, 2006; Raz et al., 2005).

If this interpretation is correct, we may speculate that the abundant encoding of irrelevant information may lead to a greater need for inhibitory control operations at later processing stages. This hypothesis is consistent with the load-shift model of executive functioning suggested by Velanova et al. (2007). In this model, younger adults rely on a combination of top-down mediated earlyand late selection processes. Older adults may be characterized by an early selection deficit in terms of unconstrained processing, leading to more effortful processing of less distinct representations at later stages. This additional need for processing at later stages may add to, or interact with, the breakdown of early inhibition processes, especially when WM capacity is taxed.

\section{Conclusion}

In line with previous research, we observed larger alpha power ipsilateral to the attended hemifield during the retention period in younger adults. Furthermore, age group differences in alpha power lateralization were load-dependent. Similar to younger adults, children and older adults showed an increase in lateralization alpha power from low to medium load, demonstrating that inhibitory processing in the service of attention regulation is present in childhood and old age. However, under conditions that highly taxed WM capacity, the lateralization of alpha power disappeared in children and older adults, pointing to difficulties in sustaining inhibition of the unattended hemisphere at high load. This effect was not due to differences in relative task difficulty between age groups. Furthermore, older adults showed higher inter-trial phase stability of alpha activity shortly after stimulus presentation, probably indicating a stronger entrainment by external stimulation leading to abundant information encoding. This effect was unique for the group of older adults. The present study went beyond the apparent symmetry in the behavioral development of WM capacity from childhood to old age, and provided initial evidence that the oscillatory mechanisms associated with lower WM performance in children and older adults relative to younger adults show both commonalities and differences.

\section{Acknowledgments}

This study was conducted within the project, "Cognitive and Neuronal Dynamics of Memory across the Lifespan (CONMEM)" at the Center for Lifespan Psychology, Max Planck Institute for Human Development, Berlin, Germany. The Max Planck Society and the German Research Foundation (DFG, HE 3347/3-1) financially supported the research. The study was conducted in partial fulfillment of the doctoral dissertation of MCS. MCS expresses gratitude to the support by the graduate program Berlin School of Mind and Brain, Humboldt Universität zu Berlin, Germany. We thank Berndt Wischnewski for help with programming of the experiment. We further thank all our student assistants for their support in data collection, Johanna Ernst and Eva Karduck for helping with the preprocessing of the data, and the participants for their cooperation. Special thanks go to Roman Freunberger, Yee Lee Shing, Thomas Grandy and Yana Fandakova for valuable discussions.

\section{References}

Backman, L., Ginovart, N., Dixon, R.A., Wahlin, T.B., Wahlin, A., Halldin, C., Farde, L 2000. Age-related cognitive deficits mediated by changes in the striatal dopamine system. Am. J. Psychiatry 157, 635-637.

Backman, L., Nyberg, L., Lindenberger, U., Li, S.C., Farde, L., 2006. The correlative triad among aging, dopamine, and cognition: current status and future prospects. Neurosci. Biobehav. Rev. 30, 791-807.

Brickenkamp, R., 1994. Test d2: Aufmerksamkeits-Belastungs-Test [Attention test] Hogrefe, Göttingen, Germany.

Campbell, K.L., Hasher, L., Thomas, R.C., 2010. Hyper-binding: a unique age effect. Psychol. Sci. 21, 399-405.

Cantero, J.L., Atienza, M., Cruz-Vadell, A., Suarez-Gonzalez, A., Gil-Neciga, E., 2009. Increased synchronization and decreased neural complexity underlie thalamocortical oscillatory dynamics in mild cognitive impairment. Neurolmage 46, 938-948.

Ciesielski, K.T., Ahlfors, S.P., Bedrick, E.J., Kerwin, A.A., Hamalainen, M.S., 2010. Topdown control of MEG alpha-band activity in children performing Categorical NBack Task. Neuropsychologia 48, 3573-3579.

Cowan, N., 2001. The magical number 4 in short-term memory: a reconsideration of mental storage capacity. Behav. Brain Sci. 24, 87-114 discussion 114-185. 
Cowan, N., Naveh-Benjamin, M., Kilb, A., Saults, J.S., 2006. Life-span development of visual working memory: when is feature binding difficult? Dev. Psychol. 42, 1089-1102.

Davidson, M.C., Amso, D., Anderson, L.C., Diamond, A., 2006. Development of cognitive control and executive functions from 4 to 13 years: evidence from manipulations of memory, inhibition, and task switching. Neuropsychologia 44, 2037-2078.

Dempster, F.N., 1992. The rise and fall of the inhibitory mechanism: toward a unified theory of cognitive development and aging. Dev. Rev. 12, 45-75.

D'Esposito, M., 2007. From cognitive to neural models of working memory. Philos. Trans. R. Soc. Lond. B. Biol. Sci. 362, 761-772.

Doesburg, S.M., Herdman, A.T., Ribary, U., Cheung, T., Moiseev, A., Weinberg, H., Liotti, M., Weeks, D., Grunau, R.E., 2010. Long-range synchronization and local desynchronization of alpha oscillations during visual short-term memory retention in children. Exp. Brain Res. 201, 719-727.

Freunberger, R., Fellinger, R., Sauseng, P., Gruber, W., Klimesch, W., 2009. Dissociation between phase-locked and nonphase-locked alpha oscillations in a working memory task. Hum. Brain. Mapp. 30, 3417-3425.

Freunberger, R., Werkle-Bergner, M., Griesmayr, B., Lindenberger, U., Klimesch, W., 2011. Brain oscillatory correlates of working memory constraints. Brain Res. 1375, 93-102.

Frodl, T., Meisenzahl, E.M., Muller, D., Leinsinger, G., Juckel, G., Hahn, K., Moller, H.J., Hegerl, U., 2001. The effect of the skull on event-related P300. Clin. Neurophysiol. 112, 1773-1776.

Gathercole, S.E., 1999. Cognitive approaches to the development of short-term memory. Trends Cogn. Sci. 3, 410-419.

Gazzaley, A., Clapp, W., Kelley, J., McEvoy, K., Knight, R.T., D'Esposito, M., 2008. Agerelated top-down suppression deficit in the early stages of cortical visual memory processing. Proc. Natl. Acad. Sc.i U. S. A. 105, 13122-13126.

Geigy, J.R., 1977. Scientific Tables. J.R. Geigy AG, Basel, Switzerland.

Gomarus, H.K., Althaus, M., Wijers, A.A., Minderaa, R.B., 2006. The effects of memory load and stimulus relevance on the EEG during a visual selective memory search task: an ERP and ERD/ERS study. Clin. Neurophysiol. 117, 871-884.

Hanslmayr, S., Klimesch, W., Sauseng, P., Gruber, W., Doppelmayr, M., Freunberger, R Pecherstorfer, T., 2005. Visual discrimination performance is related to decreased alpha amplitude but increased phase locking. Neurosci. Lett. 375, 64-68.

Hasher, L., Zacks, R.T., 1988. Working memory, comprehension, and aging: a review and a new view. In: Bower, G.H. (Ed.), The Psychology of Learning and Motivation: Advances in Research and Theory. Academic Press, San Diego, CA, pp. 193-225.

Hasher, L., Lustig, C., Zacks, R., 2007. Inhibitory mechanisms and the control of attention. In: Conway, A.R.A. (Ed.), Variation in Working Memory. University Press, Oxford, pp. 227-249.

Hughett, P., 2007. Accurate computation of the F-to-z and t-to-z transforms for large arguments. J. Stat. Softw. 23

Jensen, O., Mazaheri, A., 2010. Shaping functional architecture by oscillatory alpha activity: gating by inhibition. Front. Hum. Neurosci. 4, 186.

Jensen, O., Gelfand, J., Kounios, J., Lisman, J.E., 2002. Oscillations in the alpha band (9$12 \mathrm{~Hz}$ ) increase with memory load during retention in a short-term memory task. Cereb Cortex 12, 877-882.

Jost, K., Bryck, R.L., Vogel, E.K., Mayr, U., 2011. Are old adults just like low working memory young adults? Filtering efficiency and age differences in visual working memory. Cereb. Cortex. 21, 1147-1154.

Jung, T.P., Makeig, S., Humphries, C., Lee, T.W., McKeown, M.J., Iragui, V., Sejnowski, T.J., 2000. Removing electroencephalographic artifacts by blind source separation. Psychophysiology 37, 163-178.

Kelly, S.P., Lalor, E.C., Reilly, R.B., Foxe, J.J., 2006. Increases in alpha oscillatory power reflect an active retinotopic mechanism for distracter suppression during sustained visuospatial attention. J. Neurophysiol. 95, 3844-3851.

Klimesch, W., 1999. EEG alpha and theta oscillations reflect cognitive and memory performance: a review and analysis. Brain Res. Brain Res. Rev. 29, 169-195.

Klimesch, W., Schack, B., Schabus, M., Doppelmayr, M., Gruber, W., Sauseng, P., 2004 Phase-locked alpha and theta oscillations generate the P1-N1 complex and are related to memory performance. Brain Res. Cogn. Brain. Res. 19, 302-316.

Klimesch, W., Sauseng, P., Hanslmayr, S., 2007a. EEG alpha oscillations: the inhibitiontiming hypothesis. Brain Res. Rev. 53, 63-88.

Klimesch, W., Sauseng, P., Hanslmayr, S., Gruber, W., Freunberger, R., 2007b. Eventrelated phase reorganization may explain evoked neural dynamics. Neurosci. Biobehav. Rev. 31, 1003-1016.

Kray, J., Lindenberger, U., 2000. Adult age differences in task switching. Psychol. Aging $15,126-147$.

Lavie, N., Hirst, A., de Fockert, J.W., Viding, E., 2004. Load theory of selective attention and cognitive control. J. Exp. Psychol. Gen. 133, 339-354.

Lehrl, S., 1977. Mehrfachwahl-Wortschatz-Test b (MWT-b) [Multiplechoice vocabulary test]. Straube, Erlangen, Germany.

Li, S.-C., von Oertzen, Timo, Lindenberger, Ulman, 2006. A neurocomputational model of stochastic resonance and aging. Neurocomputing 69, 1553-1560.

Lindenberger, U., Burzynska, A.Z., Nagel, I.E., in press. Heterogeneity in frontal-lobe aging. In: Stuss, D.T., Knight, R.T. (Eds.), Principles of frontal lobe functions. Oxford University Press, New York.

Luck, S.J., Vogel, E.K., 1997. The capacity of visual working memory for features and conjunctions. Nature 390, 279-281.

MacDonald, S.W., Nyberg, L., Backman, L., 2006. Intra-individual variability in behavior: links to brain structure, neurotransmission and neuronal activity. Trends Neurosci. 29, 474-480.

McNab, F., Klingberg, T., 2008. Prefrontal cortex and basal ganglia control access to working memory. Nat. Neurosci. $11,103-107$.

Miller, G.A., 1956. The magical number seven, plus or minus two: some limits on our capacity for processing information. Psychol. Rev. 63.
Müller, V., Gruber, W., Klimesch, W., Lindenberger, U., 2009. Lifespan differences in cortical dynamics of auditory perception. Dev. Sci. 12, 839-853.

Nagel, I.E., Preuschhof, C., Li, S.C., Nyberg, L., Backman, L., Lindenberger, U., Heekeren, H.R., 2009. Performance level modulates adult age differences in brain activation during spatial working memory. Proc. Natl. Acad. Sci. U. S. A. 106, 22552-22557.

Park, D.C., Payer, D., 2006. Working memory across the adult lifespan. In: Bialystok, E., Craik, F.I.M. (Eds.), Lifespan Cognition: Mechanisms of Change. Oxford University Press, New York, pp. 128-142.

Park, D.C., Polk, T.A., Park, R., Minear, M., Savage, A., Smith, M.R., 2004. Aging reduces neural specialization in ventral visual cortex. Proc. Natl. Acad. Sci. U. S. A. 101, 13091-13095.

Raz, N., Rodrigue, K.M., 2006. Differential aging of the brain: patterns, cognitive correlates and modifiers. Neurosci. Biobehav. Rev. 30, 730-748.

Raz, N., Lindenberger, U., Rodrigue, K.M., Kennedy, K.M., Head, D., Williamson, A., Dahle, C., Gerstorf, D., Acker, J.D., 2005. Regional brain changes in aging healthy adults: general trends, individual differences and modifiers. Cereb Cortex 15, 1676-1689.

Riggs, K.J., McTaggart, J., Simpson, A., Freeman, R.P., 2006. Changes in the capacity of visual working memory in 5- to 10-year-olds. J. Exp. Child. Psychol. 95, 18-26.

Rihs, T.A., Michel, C.M., Thut, G., 2007. Mechanisms of selective inhibition in visual spatial attention are indexed by alpha-band EEG synchronization. Eur. J. Neurosci. $25,603-610$.

Rissman, J., Gazzaley, A., D'Esposito, M., 2009. The effect of non-visual working memory load on top-down modulation of visual processing. Neuropsychologia 47, 1637-1646.

Sander, M.C., Werkle-Bergner, M., Lindenberger, U., 2011a. Binding and strategic selection in working memory: a lifespan dissociation. Psychol. Aging. Advance online publication. doi:10.1037/a0023055.

Sander, M.C., Werkle-Bergner, M., Lindenberger, U., 2011b. Contralateral delay activity reveals life-span age differences in top-down modulation of working memory contents. Cereb. Cortex. Advance online publication. doi:10.1093/cercor/bhr076.

Sauseng, P., Klimesch, W., 2008. What does phase information of oscillatory brain activity tell us about cognitive processes? Neurosci. Biobehav. Rev. 32, 1001-1013.

Sauseng, P., Klimesch, W., Heise, K.F., Gruber, W.R., Holz, E., Karim, A.A., Glennon, M., Gerloff, C., Birbaumer, N., Hummel, F.C., 2009. Brain oscillatory substrates of visual short-term memory capacity. Curr. Biol. 19, 1846-1852.

Schmitz, T.W., Cheng, F.H., De Rosa, E., 2010. Failing to ignore: paradoxical neural effects of perceptual load on early attentional selection in normal aging. J. Neurosci. 30, $14750-14758$.

Schneider-Garces, N.J., Gordon, B.A., Brumback-Peltz, C.R., Shin, E., Lee, Y., Sutton, B.P., Maclin, E.L., Gratton, G., Fabiani, M., 2010. Span, CRUNCH, and beyond: working memory capacity and the aging brain. J. Cogn. Neurosci. 22, 655-669.

Segalowitz, S.J., Davies, P.L., 2004. Charting the maturation of the frontal lobe: an electrophysiological strategy. Brain Cogn. 55, 116-133.

Shing, Y.L., Werkle-Bergner, M., Li, S.C., Lindenberger, U., 2008. Associative and strategic components of episodic memory: a life-span dissociation. J. Exp. Psychol. Gen. 137, 495-513.

Sowell, E.R., Thompson, P.M., Holmes, C.J., Jernigan, T.L., Toga, A.W., 1999. In vivo evidence for post-adolescent brain maturation in frontal and striatal regions. Nat. Neurosci. 2, 859-861.

Sowell, E.R., Peterson, B.S., Thompson, P.M., Welcome, S.E., Henkenius, A.L., Toga, A.W., 2003. Mapping cortical change across the human life span. Nat. Neurosci. 6, 309-315.

Tallon-Baudry, C., Bertrand, O., Delpuech, C., Permier, J., 1996. Stimulus specificity of phase-locked and non-phase-locked $40 \mathrm{~Hz}$ visual responses in human. J. Neurosci. $16,4240-4249$

Tallon-Baudry, C., Bertrand, O., Delpuech, C., Permier, J., 1997. Oscillatory gamma-band $(30-70 \mathrm{~Hz})$ activity induced by a visual search task in humans. J. Neurosci. 17, 722-734.

Thaler, D.S., 2002. Design for an aging brain. Neurobiol. Aging 23, 13-15.

Thut, G., Nietzel, A., Brandt, S.A., Pascual-Leone, A., 2006. Alpha-band electroencephalographic activity over occipital cortex indexes visuospatial attention bias and predicts visual target detection. J. Neurosci. 26, 9494-9502.

Toga, A.W., Thompson, P.M., Sowell, E.R., 2006. Mapping brain maturation. Trends Neurosci. 29, 148-159.

Tuladhar, A.M., ter Huurne, N., Schoffelen, J.M., Maris, E., Oostenveld, R., Jensen, O., 2007. Parieto-occipital sources account for the increase in alpha activity with working memory load. Hum. Brain Mapp. 28, 785-792.

Uhlhaas, P.J., Roux, F., Rodriguez, E., Rotarska-Jagiela, A., Singer, W., 2010. Neural synchrony and the development of cortical networks. Trends Cogn. Sci. 14, 72-80.

Velanova, K., Lustig, C., Jacoby, L.L., Buckner, R.L., 2007. Evidence for frontally mediated controlled processing differences in older adults. Cereb Cortex 17, 1033-1046.

Vinck, M., van Wingerden, M., Womelsdorf, T., Fries, P., Pennartz, C.M., 2010. The pairwise phase consistency: a bias-free measure of rhythmic neuronal synchronization. Neurolmage 51, 112-122.

Vogel, E.K., Machizawa, M.G., 2004. Neural activity predicts individual differences in visual working memory capacity. Nature 428, 748-751.

Vogel, E.K., McCollough, A.W., Machizawa, M.G., 2005. Neural measures reveal individual differences in controlling access to working memory. Nature 438, 500-503.

Wechsler, D., 1955. Wechsler Adult Intelligence Scale Manual. Psychological Corporation, New York.

Werkle-Bergner, M., Shing, Y.L., Müller, V., Li, S.C., Lindenberger, U., 2009. EEG gamma-band synchronization in visual coding from childhood to old age: evidence from evoked power and inter-trial phase locking. Clin. Neurophysiol. 120, 1291-1302.

Werkle-Bergner, M., Freunberger, R., Sander, M.C., Klimesch, W., 2010. Age differences in controlling the contents of working memory: evidence from phase and amplitude measures of alpha oscillations. Poster Presented at the 17th Annual 
Meeting of the Cognitive Neuroscience Society (CNS), Montreal, Canada, April, 17th-20th, 2010.

Worden, M.S., Foxe, J.J., Wang, N., Simpson, G.V., 2000. Anticipatory biasing of visuospatial attention indexed by retinotopically specific alpha-band electroencephalography increases over occipital cortex. J. Neurosci. 20, RC63.

Yordanova, J., Kolev, V., 1997. Alpha response system in children: changes with age. Int. J. Psychophysiol. 26, 411-430.
Zanto, T.P., Gazzaley, A., 2009. Neural suppression of irrelevant information underlies optimal working memory performance. J. Neurosci. 29, 3059-3066.

Zanto, T.P., Toy, B., Gazzaley, A., 2009. Delays in neural processing during working memory encoding in normal aging. Neuropsychologia 48, 13-25.

Zanto, T.P., Hennigan, K., Ostberg, M., Clapp, W.C., Gazzaley, A., 2010. Predictive knowledge of stimulus relevance does not influence top-down suppression of irrelevant information in older adults. Cortex 46, 564-574. 